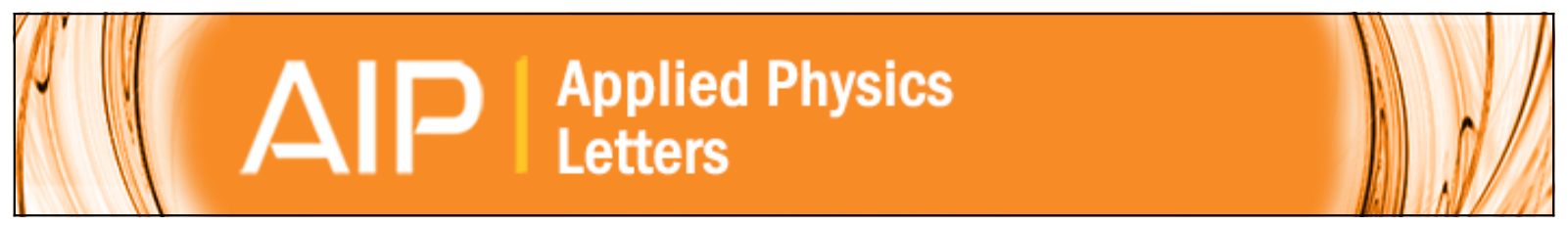

Magnetization reversal, asymmetry, and role of uncompensated spins in perpendicular exchange coupled systems

J. Camarero, J. Miguel, J. B. Goedkoop, J. Vogel, F. Romanens, S. Pizzini, F. Garcia, J. Sort, B. Dieny, and N.

B. Brookes

Citation: Applied Physics Letters 89, 232507 (2006); doi: 10.1063/1.2402882

View online: http://dx.doi.org/10.1063/1.2402882

View Table of Contents: http://scitation.aip.org/content/aip/journal/apl/89/23?ver=pdfcov

Published by the AIP Publishing 


\title{
Magnetization reversal, asymmetry, and role of uncompensated spins in perpendicular exchange coupled systems
}

\author{
J. Camarero ${ }^{a)}$ \\ Departamento de Física de la Materia Condensada, Universidad Autónoma de Madrid, 28049 Madrid, \\ Spain \\ J. Miguel ${ }^{\text {b) }}$ and J. B. Goedkoop \\ Van der Waals-Zeeman Institute, University of Amsterdam, 1018 XE Amsterdam, The Netherlands \\ J. Vogel, F. Romanens, and S. Pizzini \\ Laboratoire Louis Neel, CNRS, 25 avenue des Martyrs, BP 166, F-38042 Grenoble Cedex 9, France \\ F. Garcia, ${ }^{\mathrm{c})}$ J. Sort, ${ }^{\mathrm{d})}$ and B. Dieny \\ SPINTEC (URA2512 CNRS/CEA), CEA-Grenoble, 38054 Grenoble Cedex 9, France \\ N. B. Brookes \\ European Synchrotron Radiation Facility (ESRF), 38043 Grenoble, France
}

(Received 16 August 2006; accepted 25 October 2006; published online 6 December 2006)

\begin{abstract}
Soft $\mathrm{x}$-ray resonant magnetic scattering has been used to investigate the element-selective microscopic magnetization reversal behavior of room temperature perpendicular exchange coupled ferromagnetic/antiferromagnetic (F/AF) systems and to study the role of the interfacial coupling strength on it. Different nucleation processes and domain size distributions along the decreasing and increasing branches of the reversal have been found. The size of the magnetic domains during reversal depends on both the F anisotropy and F/AF coupling strength, decreasing when one of them increases. Evidence of the exchange bias (coercivity enhancement) being induced by pinned (unpinned) uncompensated AF interfacial spins is also shown. (C) 2006 American Institute of Physics. [DOI: 10.1063/1.2402882]
\end{abstract}

The interfacial exchange coupling in ferromagnetic/ antiferromagnetic (F/AF) systems is being extensively studied both for the key role it plays in spintronic devices and for its interesting basic properties. The local character of the phenomenon was correctly described in the original publication, ${ }^{1}$ and it has been visualized recently at the atomic scale. ${ }^{2}$ The most notable changes in the $\mathrm{F}$ hysteresis loops are a shift away from the zero field axis, called exchange bias $H_{E}$, an enhanced coercivity $\Delta H_{C}$, and a change of the overall magnetization reversal of the $\mathrm{F}$ layer. ${ }^{3}$ The former features are actually used in advanced magnetic in-plane spintronic devices, by pinning the harder reference layer in spin valve heads and magnetic random access memory circuits, and will play an important role in future nanometer-sized magnetic devices, by effectively increasing the stability of magnetic nanostructures. ${ }^{2,4}$ Future advances are promoted by the recent observation of perpendicular exchange-coupling effects in several F/AF systems below ${ }^{5-7}$ and above room temperature. $^{8-10}$

Most of the studies on F/AF systems have focused on in-plane magnetization systems. Recently, it has been found that the pinned and unpinned uncompensated AF spins at the interface are correlated with $H_{E}$ (Ref. 11) $\Delta H_{C}$ (Ref. 12), respectively, and that the competition between anisotropies

\footnotetext{
${ }^{a)}$ Electronic mail: julio.camarero@uam.es.

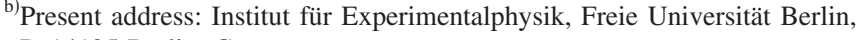
D-14195 Berlin, Germany.

${ }^{c}$ Present address: Brazilian Synchrotron Light Laboratory, LNLS, CEP 13084-971, Campinas, SP, Brazil.

d) Present address: ICREA and Departament de Fìsica, Universitat Autónoma de Barcelona, 08193 Bellaterra, Spain.
}

determines the asymmetric behavior of the magnetization reversal. $^{13}$

In this letter we report on all of the aforementioned unusual magnetic properties but in room temperature perpendicular exchange coupled $[\operatorname{Pt}(2.3 \mathrm{~nm}) / \mathrm{Co}(0.4 \mathrm{~nm})]_{n} / 10 \mathrm{~nm}$ FeMn films studied by means of soft x-ray resonant magnetic scattering (SXRMS). The preparation and structural characterization of the films as well as the SXRMS technique are described in the supporting information. ${ }^{14}$ The study has been performed on two samples, with $n=10$ and 15, which present different effects of the F/AF interface coupling. Enhanced coercivity is present in both samples, but there is exchange bias only for the $n=10$ sample, which has a smaller number of repeats, i.e., smaller effective $\mathrm{F}$ thickness. ${ }^{8}$

Figure 1(a) shows typical $\mathrm{x}$-ray absorption spectra (XAS) and the corresponding dichroism spectra [X-ray magnetic circular dichroism (XMCD)] recorded in transmission geometry at the Fe and Co $L_{3,2}$ absorption edges for the $n$ $=15$ sample. The presence of XMCD signal at the Fe $L_{3,2}$ edges clearly shows the existence of uncompensated Fe moments at the $\mathrm{Co} / \mathrm{FeMn}$ interface. The XMCD curve has a maximum asymmetry (i.e., dichroism divided by the helicity averaged absorption) of about $3.1 \%$ at the $\mathrm{Fe} L_{3}$ white line. We estimate a nominal thickness of uncompensated Fe moments of $1.8 \pm 0.4(1.4 \pm 0.4)$ ML of FeMn for $n=15(n=10)$. These values are somewhat larger than the ones obtained for in-plane systems, ${ }^{11,12}$ which would indicate larger interdiffusion at the F/AF interface. In addition, the sign of the XMCD signals reveals a parallel alignment between the Co and the uncompensated Fe moments.

Element-selective hysteresis loops were obtained by measuring the absorption signal as a function of applied 

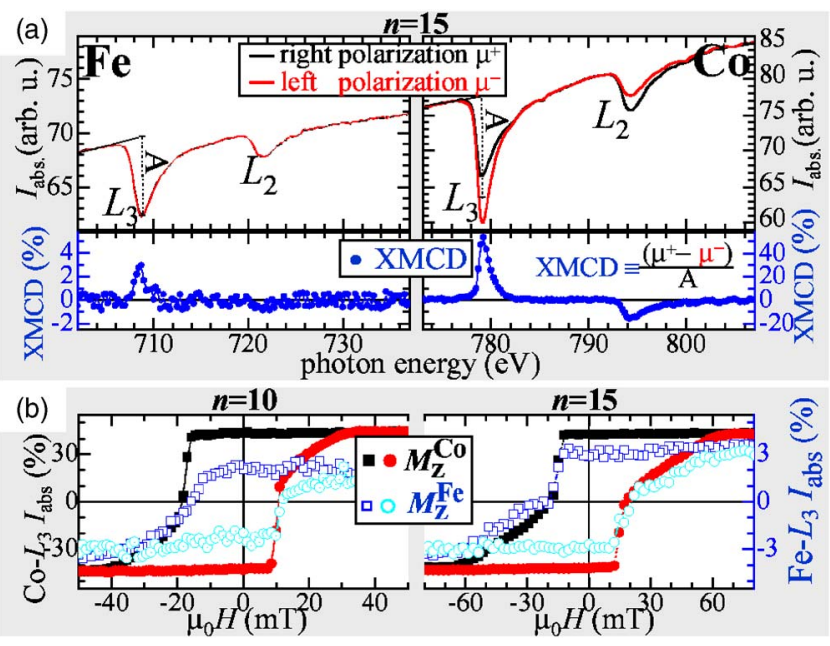

FIG. 1. (Color online) SXRMS element-selective magnetization reversal study. (a) XAS (upper curves) and XMCD (lower curves) spectra recorded at the Fe (left) and Co (right) $L_{2,3}$ edges of the sample with $n=15$ measured with a flipping field of $100 \mathrm{mT}$. (b) Element-selective hysteresis curves of the sample with $n=10$ (left column) and $n=15$ (right column). The filled symbols and the left $Y$-axis scale correspond to the $\mathrm{F}$ layer, whereas the open symbols and the right $Y$-axis scale correspond to the AF layer.

field, at a fixed energy corresponding to the maximum XMCD signal. The field dependence of the Co $L_{3}$ absorption intensity shown in Fig. 1(b) (filled symbols) reproduces the magnetization hysteresis curves measured by magnetooptical Kerr effect. The interface exchange coupling leads to an enhancement of the coercive field, from $5 \mathrm{mT}$ in the same $\mathrm{Pt} / \mathrm{Co}$ multilayer without $\mathrm{AF}$ layer to about $12.5 \mathrm{mT}$ $(18.5 \mathrm{mT})$, as well as to an exchange bias field of $4.5 \mathrm{mT}$ $(0 \mathrm{mT})$ for the sample with $n=10(n=15)$. The field dependence of the $\mathrm{Fe} L_{3}$ absorption intensity [empty symbols in Fig. 1(b)] indicates the presence of uncompensated AF spins, aligned parallel to the $\mathrm{F}$ spins, which are dragged during the reversal of the $\mathrm{F}$ layer. The unpinned uncompensated $\mathrm{AF}$ spins have been taken as responsible for the $H_{C}$ enhancement in in-plane exchange F/AF systems. ${ }^{12}$ Note that the amount of uncompensated AF spins that are dragged during the reversal of the $\mathrm{F}$ layer is larger for the sample with $n=15$. The dichroic asymmetry is proportional to the number of reversed ferromagnetic spins. Assuming a similar spin structure at the F/AF interface (both samples were grown in the same run), the same density of uncompensated spins in the two samples is expected. For the $n=15$ sample all the uncompensated spins are dragged during the $\mathrm{F}$ reversal. $H_{E}$ $=0$ and only $H_{C}$ enhancement is hence observed. For the $n$ $=10$ sample, about $60 \%$ of the total amount of uncompensated AF spins is dragged during the F reversal, causing the coercivity enhancement. The remaining $40 \%$ of the AF spins stays pinned, and this is the part that causes the exchange bias. Similar findings have been reported in in-plane exchange coupled F/AF systems. ${ }^{11}$

The field-dependent total scattered intensity loops $I_{\text {scatt }}(H)$ are shown in the middle graphs of Fig. 2. In general, the two samples show similar features, which can be easily correlated with the hysteresis curves $I_{\text {abs }}(H)$ (upper graphs of Fig. 2). Magnetic scattering originates from deviations from uniform magnetization, and is therefore extremely sensitive to the nucleation processes at the onset of magnetization reversal. In this context, for large fields, the $\mathrm{F}$ layer is uniformly magnetized and gives no contribution to $I_{\text {scatt }}$. At the

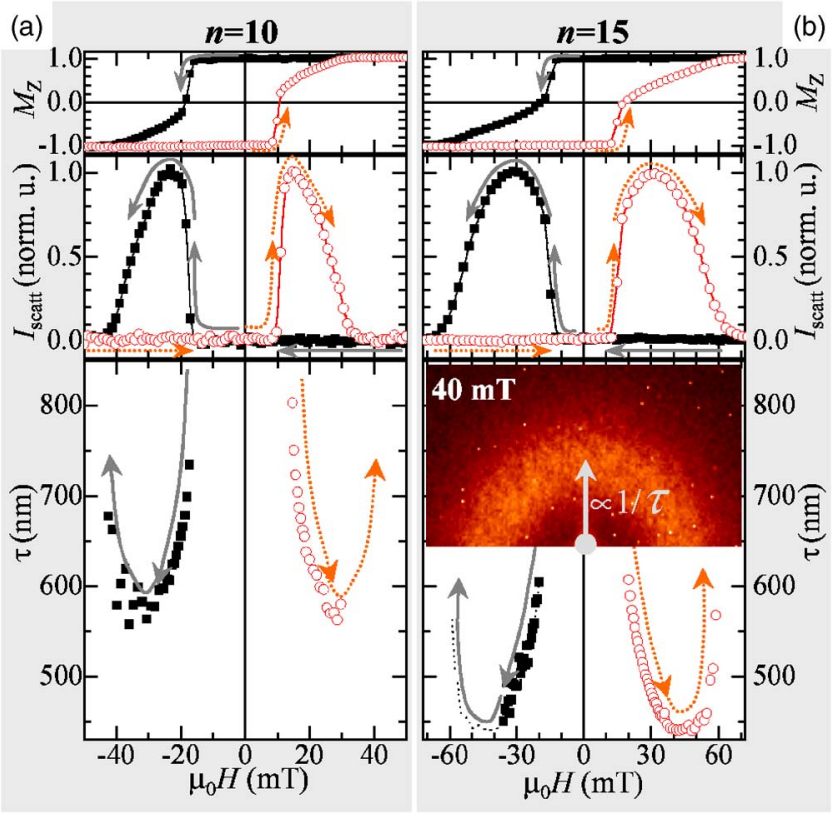

FIG. 2. (Color online) SXRMS microscopic magnetization reversal study of (a) the sample with $n=10$, which shows both exchange bias and coercivity enhancement, and (b) the sample with $n=15$, which only shows coercivity enhancement. Field-dependent magnetization (upper graphs), total scattering intensity $I_{\text {scatt }}$ over all the $q$ space available with the diffractometer $(<1 \mu \mathrm{m})$ (middle graphs), and magnetic domain period $\tau$ (bottom graphs) measured at $\mathrm{Co} L_{3}$. The domain period $\tau$ was extracted from $2 \mathrm{D} q$-space resolved images measured during the reversal, as shown the inset of the right bottom graph. The continuous (dotted) arrows are guidelines for the eye to identify the evolution of the decreasing (increasing) reversal branch.

nucleation point, i.e., at the onset of magnetization reversal, $I_{\text {scatt }}$ increases. The slope of this increase gets smaller when the reversal transition becomes slower, and $I_{\text {scatt }}$ reaches a maximum roughly at the middle of this slower transition. The scattered intensity then decreases monotonously up to the saturation field. This behavior is consistent with magnetization reversal proceeding through nucleation, propagation, and annihilation of magnetic domains.

The microscopic information of the reversal process is obtained by measuring two-dimensional (2D) $q$-space resolved images as a function of applied field, like the image depicted in the inset of the bottom graph in Fig. 2(b). The ring shape of the scattered signal, also observed for the $n$ $=10$ sample (not shown), indicates that a randomly oriented magnetic domain structure is formed during the reversal. In this case, an average distance between domains (domain period) of $\tau \simeq 480 \mathrm{~nm}$ is extracted from the separation between this ring and the transmitted beam. The evolution of the domain period extracted from the 2D images is depicted in the bottom graphs of Fig. 2. The curves show a quasiparabolic shape: close to nucleation and saturation the period diverges to values above $1 \mu \mathrm{m}$, corresponding to the small $q$ limit set by the beam stop. In particular, smaller domain period and therefore smaller domain size are observed for the sample with more $[\mathrm{Co} / \mathrm{Pt}]$ periods, i.e., the $n=15$ sample. In $\mathrm{F}$ thin films with perpendicular magnetic anisotropy, the low dimensionality causes the domain sizes in the demagnetized state to be small in order to minimize the dipolar energy. ${ }^{15}$ Since the domain size is determined by the balance between the gain in magnetostatic energy realized by the formation of domains versus the cost of creating domain walls, the difference in domain size is likely to be due to the stronger mag- 


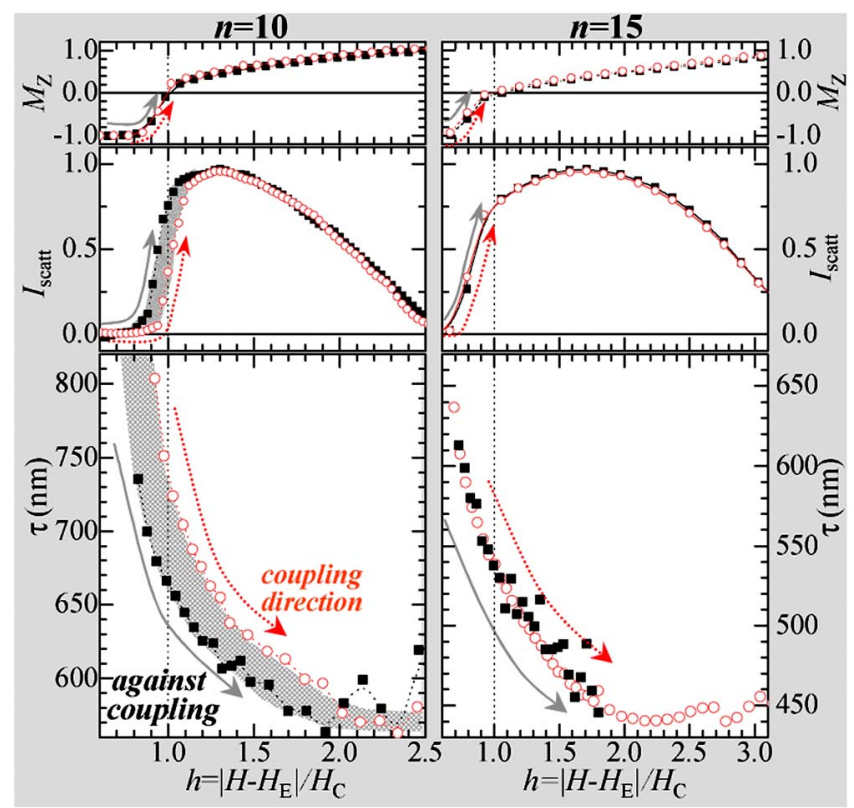

FIG. 3. (Color online) Exchange-coupling dependent magnetization reversal asymmetry. Field-dependent magnetization (upper graphs), total scattering intensity $I_{\text {scatt }}$ (middle graphs), and magnetic domain period $\tau$ (bottom graphs) measured at Co $L_{3}$. The data shown on Fig. 2 have been corrected for the average bias field $H_{E}$ and then plotted vs reduced field $h=\mid H$ $-H_{E} \mid / H_{C}$ to compare the decreasing (filled squares, against coupling) and increasing (open circles, coupling direction) magnetization branches.

netization and therefore dipolar interaction for the $n=15$ sample.

Although the reversal in both branches of the hysteresis takes place by nucleation and further propagation, a clear difference in the nucleation behavior during $\mathrm{F}$ reversal is observed for the sample with $H_{E} \neq 0$, i.e., $n=10$. In Fig. 3, the data shown in Fig. 2 are corrected for the average bias value and then plotted versus the reduced field value $h=\mid H$ $-H_{E} / / H_{C}$. The magnetization $M_{Z}(h)$ (upper graph) shows identical behavior in both branches. The integrated scattering intensity $I_{\text {scatt }}(h)$ [middle graph of Fig. 3(a)] shows a different initial nucleation behavior for the two reversal branches but once $h>1.2$ the reversal appears identical in both branches. A less pronounced but similar feature was previously reported by Hellwig et al. in perpendicular exchange coupled $\left[[\mathrm{Co} / \mathrm{Pt}]_{5} / \mathrm{CoO}\right]_{10}$ multilayers. ${ }^{7}$ The authors claim that this feature is in contrast to many in-plane biased experiments where an asymmetry during the magnetization reversal appears to be a rather general property. However, the bottom graph of Fig. 3(a) shows a clear asymmetry during reversal. The domain period, and therefore the domain size during magnetization reversal, is smaller for the decreasing branch, where the field is applied against the exchange coupling direction. For the same reason, the density of $\mathrm{F}$ domains during reversal is larger when the field is applied antiparallel to the exchange bias field direction, like in in-plane exchange-biased systems. ${ }^{16}$ This asymmetric behavior has also been shown by Kerr microscopy measurements in perpendicular exchange-biased $[\mathrm{Pt} / \mathrm{Co}]_{\mathrm{n}} / \mathrm{IrMn}$ films. ${ }^{10}$
In summary, we have investigated the microscopic magnetization reversal behavior of room temperature perpendicular exchange coupled $[\mathrm{Pt} / \mathrm{Co}]_{\mathrm{n}} / \mathrm{FeMn}$ films by means of SXRMS. The element selectivity reveals a parallel alignment between the $\mathrm{F}$ and the uncompensated AF moments, as well as the influence of the pinned (unpinned) uncompensated $\mathrm{AF}$ spins on $H_{E}\left(\Delta H_{C}\right)$. The magnetization reversal is characterized by nucleation, propagation, and annihilation of magnetic domains. The F domain size depends on the relative size of the different anisotropy terms (perpendicular, dipolar, unidirectional) and on the direction of the applied magnetic field. When $H_{E} \neq 0$, different nucleation processes and domain size distributions are found when the field is applied antiparallel or parallel to the exchange coupling direction. Our fundamental findings are of great importance for the development of future spintronic devices with perpendicular magnetization with custom-chosen properties.

One of the authors (J.C.) acknowledges support through a "Ramón y Cajal" contract from the Spanish MEC. This research was partly supported by the CAM through Project No. GR/MAT/0153/2004 and is also a part of the research program of the Stichting voor Fundamenteel Onderzoek der Materie (FOM) with financial support from the Nederlandse Organisatie voor Wetenschappelijk Onderzoek (NWO). Two of the authors (J.C. and J.V.) acknowledge partial financial support for personnel exchange by the "Acciones IntegradasPicasso" Programme, through Grant No. HF2003-0173. The authors thank K. Larsson (ESRF) for his support.

${ }^{1}$ W. H. Meiklejohn and C. P. Bean, Phys. Rev. 102, 1413 (1956).

${ }^{2}$ J. Camarero, A. L. Vázquez de Parga, T. K. Yamada, T. Mizoguchi, and H. van Kempen (unpublished).

${ }^{3}$ J. Nogués and I. K. Schuller, J. Magn. Magn. Mater. 192, 203 (1999).

${ }^{4}$ V. Skumryev, S. Stoyanov, Y. Zhang, G. Hadjipanayis, D. Givord, and J. Nogués, Nature (London) 423, 850 (2003).

${ }^{5}$ B. Kagerer, Ch. Binek, and W. Kleemann, J. Magn. Magn. Mater. 217, 139 (2000).

${ }^{6}$ S. Maat, K. Takano, S. S. P. Parkin, and E. E. Fullerton, Phys. Rev. Lett. 87, 087202 (2001).

${ }^{7}$ O. Hellwig, S. Maat, J. B. Kortright, and Eric E Fullerton, Phys. Rev. B 65, 144418 (2002).

${ }^{8}$ F. Garcia, G. Casali, S. Auffret, B. Rodmacq, and B. Dieny, J. Appl. Phys. 91, 6905 (2002)

${ }^{9}$ F. Garcia, J. Moritz, F. Ernult, S. Aufret, B. Rodmacq, B. Dieny, J. Camarero, Y. Pennec, S. Pizzini, and J. Vogel, IEEE Trans. Magn. 38, 2730 (2002).

${ }^{10}$ F. Romanens, S. Pizzini, F. Yokaichiya, M. Bonfim, Y. Pennec, J. Camarero, J. Vogel, J. Sort, F. Garcia, B. Rodmacq, and B. Dieny, Phys. Rev. B 72, 134410 (2005).

${ }^{11}$ H. Ohldag, A. Scholl, F. Nolting, E. Arenholz, S. Maat, A. T. Young, M. Carey, and J. Stöhr, Phys. Rev. Lett. 91, 017203 (2003).

${ }^{12}$ J. Camarero, Y. Pennec, J. Vogel, S. Pizzini, M. Cartier, F. Fettar, F. Ernult, A. Tagliaferri, N. B. Brookes, and B. Dieny, Phys. Rev. B 67, 020413(R) (2003).

${ }^{13}$ J. Camarero, J. Sort, A. Hoffmann, J. M. García-Martín, B. Dieny, R. Miranda, and J. Nogués, Phys. Rev. Lett. 95, 057204 (2005).

${ }^{14}$ See EPAPS Document No. E-APPLAB-89-247649 for sample preparation and structural characterization, as well as for the description of the SXRMS technique. This document can be reached via a direct link in the online article's HTML reference section or via the EPAPS homepage (http://www.aip.org/pubservs/epaps.html).

${ }^{15}$ S.-B Choe, H.-J. Jang, and S.-C. Shin, J. Appl. Phys. 85, 5651 (1999).

${ }^{16}$ A. Kirilyuk, Th. Rasing, H. Jaffrès, D. Lacour, and F. Nguyen Van Dau, J. Appl. Phys. 91, 7745 (2002). 